\title{
Traffic Sustainable Development Research on Historical Commercial
}

\section{Blocks}

\author{
Xiance Zeng \\ ArchitecturalDesignandTheory,South China University of Technology (SCUT),Guangzhou,510641
}

Key words:Historical commercial district, the traffic system, sustainable development, streets space characteristics, green transportation, parking system

\begin{abstract}
In the history of commercial blocks, in addition to carrying commercial function of the building, another important element is the street. The spatial layout of the street, reflects the concept of urban planning and construction technology level over a specific period of time; Street connection of historic buildings or historical city area, become important clues to understand urban commercial culture context. Street space with the formation of the square, the courtyard space, together constitute the public space of the commercial block. Street traffic sustainable development, historical style and features of historical business district, commercial vitality and have significant influence on quality of space.
\end{abstract}

\section{The Definition of Historic Blocks and Historical Commercial District}

The relics and historical block, it is to point to more concentrated, or can more fully reflect a certain historical period and the local ethnic characteristics of traditional features of the blocks.[1] The concept is first proposed in 1933, the international society for modern architecture in Athens by August - in the Athens charter "of historic buildings and blocks shall be completed to save, not to destroy". And historical commercial district, is refers to from a particular historical period beginning with commercial nature and function of commercial forms as the main functions of the block, to support business activities on the basis of historic blocks, such as Beijing's qianmen street, Kuan\&zhai alley and Jinli of Chengdu, etc. The characteristics of the history of commercial block is formed by a series of individual buildings on layout group, and spread along the street, be a certain area where reflect regional development, economic level, the carrier of folk customs, regional features, etc.[2]

\section{The Relationship between Historical Commercial Blocks and Street Traffic}

Historical commercial blocks' traffic system, on the one hand, to satisfy the various groups in certain areas of travel activities and become the people entertainment and interchange activities of space; On the other hand also ensures the transportation of commercial goods. With the development of modern cities, historical commercial street originally has a history of commercial atmosphere and space is gradually replaced by urban development, the contradiction between history and modern has also been gradually create, the most prominent is the growing number of motor vehicles and historic business blocks limited traffic space form or contradiction between the traffic capacity. And historical commercial district as an important component of urban functional structure, need via the traffic system which is formed by street to connect the people and goods inside and outside. The contradiction between the limitation of the history and the demand of the 
development is still deepening.(Fig.1)

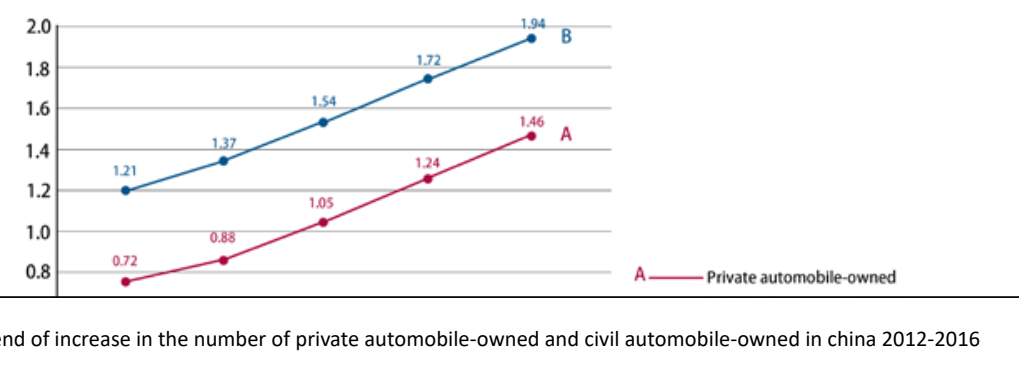

In history, human means of transportation have been processed. The earliest human transportation period, people is the main part of the street, the needed scale for street space is match with the fitting body; And with the appear of livestock transport, bullock carts and carriages compared with human transportation has greater transport capacity, make the space of the commercial streets scale have expanded. [3]And with the generate of the motorized transportation, the pedestrian traffic is gradually replaced by the traffic for vehicles, transport and subsequently change, from small car and small trucks to large truck, the increase of transport capacity is also directly lead to the growth of street space scales in historical commercial blocks.(fig.2) But the urban construction and the evolution of the mode of transport is not synchronized, the traditional street space is difficult to maintain the increasing traffic pressure.

And in order to adapt to the trend of commuting habits to change the historical commercial block scale and spatial form is also not very effective the new superseding to the old style improvement brings a certain impact, make use of every single space transformation also makes the traffic is becoming more and more crowded, road widening and straightening more intensified the destruction of the historical commercial blocks' style and features also commercial atmosphere.

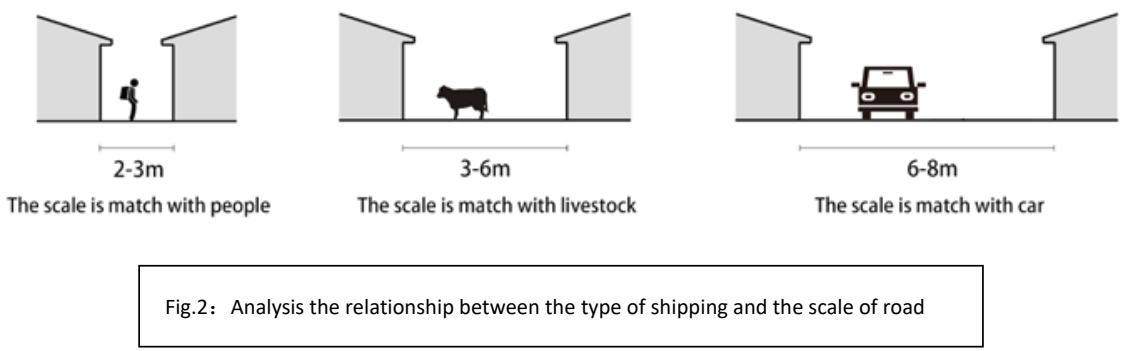

\section{The current situation and problems of historical commercial blocks}

\section{Analysis from the Angle of urban construction}

With the development of urban construction, most of the historical center area has break through the scope of the original in china. With historical urban area as the center, the pattern of development is layers expansion, historical commercial blocks also gradually be surrounded by the historical city .[4]And historical commercial blocks'social function such as commercial and residential is still in the continuing development. The transportation system and street space as part of the urban traffic network and play an important role, it also makes the contradiction between historical commercial blocks' social function of protection, the protection of traditional style and the shortage of traffic space become deeply.

Analysis from the Angle of traffic function. Due to historical commercial block roads are historical continuous form, even if it changes in developing, but still retains the primitive type, and the streets of space form, this historical continued the road system structure has been unable to meet 
the modern road traffic capacity as needed.

Historical commercial blocks intersection formed the road corner space, is often the area of the most dense commercial atmosphere. But with the influx of a large number of motor vehicles, the intersection where gathered the stream of people also become the focus of traffic from every direction.[5]If lack of traffic organization, the vehicle in the intersection position may form the congestion, leading to the historical street area traffic capacity is insufficient.

Because of the historical blocks' characteristic of business functions, to ensure the sustainable development of its function, we must guarantee the existence of its commercial activity, attract enough shopping flow became the main way of keeping commercial vitality. But as the motor vehicle replaced the way on foot, appeal to the people, also means the increase in the history of commercial blocks number of private cars. At the same time, due to the demand of commercial goods storage and transportation, a large number of freight traffic will also bring huge of traffic pressure in historical commercial blocks.

Because historic commercial blocks are the characteristics of dense layout, so it is difficult to set up the parking facilities which can matching the modern traffic capacity. Based on its business nature, often require large vehicles to enter for the loading and unloading of the goods, so a lot of storage and parking space is needed to historical commercial blocks. At the same time also need to meet the demand of commercial passenger traffic stop. And public parking facilities is insufficient, then the problem is the vehicle random stop, coupled with the lack of management measures, disorderly parked vehicle led to more congested.

Public transport is a good way to solve the traffic capacity, but in the existing historical commercial blocks, considering the characteristic of commercial activities have flow stagnation, will limit the development of public transport. Unreasonable public transportation planning and design, and the management measures for the car traffic and parking is not enough, even if the public transport is introduced in historical commercial blocks, the traffic congestion degree will also exacerbate. While walking and slow traffic are oppressed by public transportation, it is in a state of lack. The pedestrian with bicycles and cars are mixed, lack of isolation space between each other, and also made the walk or slow system development severely affected.[6]

Therefore, to maintain the social function, the blocks style and the business environment can continue in historical commercial blocks. To ensure the vitality and sustainable development of historical commercial blocks, needs with a view to the planning of urban construction, in the perspective of traffic function, from the perspective of the design, management, etc, to ensure sustainable development of the historical commercial blocks.

\section{The historical commercial blocks traffic sustainable development strategy}

The sustainable development of the historical commercial blocks traffic, requires a combination of the history of the existing urban traffic development strategy, and combined with the function of the commercial block' attribute and spatial characteristics, formulate the sustainable development of historical commercial blocks' the traffic strategy. By means of transportation, the road system, parking system, management system and the block space utilization is optimized, and combining the business format and internal function of blocks, to organize the traffic flow adjust measures to local conditions, and protection of historical commercial blocks.[7]

Balance the supply and demand of traffic, reasonable control of motor vehicle type and quantity. Due to the development of the motor vehicle traffic is the principal cause of historical commercial blocks the traffic problem. So we need to start from motor vehicle, to adjust the balance of supply and demand of traffic. Because of the limitation of historical commercial block space 
scale, the traffic capacity is often in a state of lack, and large vehicle for transportation for commercial goods will aggravate traffic burden. So on the vehicle quantity control method, the proportion of freight vehicles can be appropriately increase and reduce the proportion of private cars into the historical commercial block, guide citizens and tourists to other forms of transportation (including public traffic, slow traffic or walking) through the historical commercial blocks.

To set up appropriate traffic shielding system. Traffic shielding system, have the same function with the urban periphery beltway, to attract the traffic from interior road system to external trunk road system, and strengthen the connection between branch network and trunk road blocks, forming the cycle of sustainable transportation system, reduce internal block traffic problem caused by motor vehicles.(fig.3)

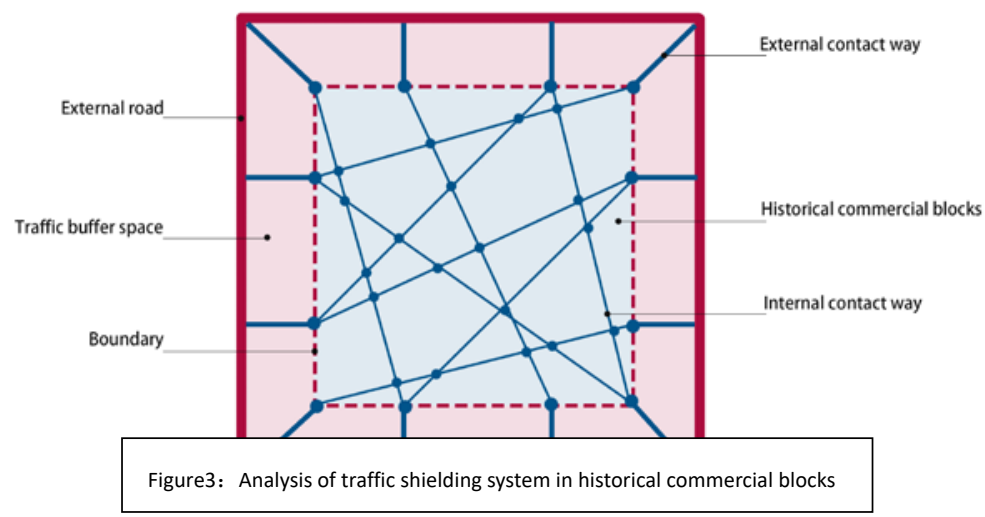

Improve street parking system, rational utilization of underground space block. Because of the limitation of spatial pattern of historical commercial block, it cannot provide large public parking space effectively, while guarantee the set up of parking space in external historical commercial blocks and advocate green transfer at the same time, can also be reasonable development of underground space, and set underground parking space in the historical commercial blocks to adjust measures to local conditions, establishing the public parking system with the blocks, to solve the parking problem.At the same time, the development of underground space also can be used as a transport of goods transit point.

Optimization of bus system, development of slow traffic, establish the green traffic system. Historical commercial blocks within small scale traffic space, cannot meet the city rail transit and larger bus demand for road space, soconsider to increase the mini bus, electric sight-seeing cars or other friendly public transport, to ensure the effectively connects with the peripheral city public traffic system.[8]Mini bus and electric cars as a tourism project, can promote historical commercial blocks tourism sustainable development. And the bike-share as a new traffic pattern, also can effectively promote the slow traffic system development, and help to establishment of the green traffic system.

Strengthen the management of street traffic. Strengthen historical commercial blocks traffic management, setting up clear indication marks in no parking area, and timely advice by designated personnel, investigate and forbid the phenomenon of disorderly stopping place. At the same time, the historical commercial blocks in accordance with the road grade, make reasonable speed limit laws and regulations, through artificial and system of management, make the commercial blocks' traffic effectively improved.

Adjust the commercial function reasonable, organize the commercial and tourism flow. Historical commercial blocks after each historical period of development, formed a comparatively complete system of commercial function. But under the influence of electricity business, centralized 
business and other modern business model, to keep the business sustainable development, will need to adjust commercial function. In the process of adjusting commercial function, need to plan reasonable business streamline, by this way to control the distribution of the traffic flow in the whole block, through methods such as replacement of function to guarantee the sustainable development of transportation.

Maintenance blocks historical style and features. Historical commercial blocks contains the content of history and culture is often through commercial the space form and historical style and features or other aspect, while adjusting and optimizing of the traffic conditions and history keep blocks traffic sustainable development, also need to retaining the historical style and features of block.

\section{Conclusion}

Historical commercial blocks traffic sustainable development, is related to business vitality and sustainable development. Therefore, to ensure the historical commercial blocks' history vitality, business vitality, and to keep its sustainable development, in the traffic level, requires the adoption of certain planning and management strategy, try to solve the problem of the traffic sustainable development. Through strengthening the construction of transportation and adopt a series of optimization measures, can make the historical commercial blocks to some extent of the traffic ability get promoted, which can also leads to the improvement of the traffic environment and sustainable development in the level of whole city.

\section{References}

[1] Li Yunhe, Huaxia Cathay’s Ideas, Tianjin university press, PP325, 2005.

[2] Ruanyisan, SunMeng, Historical block protection and planning problems of research in China ,Journal of Shanghai urban planning, (4),pp.2-9,2010

[3] Kevin lynch, The Image of the City, Huaxiapress, Beijing, pp.87, 2001.

[4] ZhuoJian, Study on the urban streets and the planning and design, China building industry press, Beijing, 309, 2010

[5] HuiYing, Traffic planning and design framework to explore the historical block, Journal of urban planning, (5), pp.101-106, 2009

[6] ZhuoJian, Slow traffic between, Journal of ideal space,(59),pp.12-15, 2013.

[7] Wen Guowei, The historic city traffic problem analysis, Journal of China city, (01), pp.33-35, 2012.

[8] Zhou Ganshi. Developmentof our country big city transportationresearch, China building industry press, Beijing, 56, 1997. 